\title{
Structural biology of prokaryotic cell surfaces
}

\author{
Tanmay Bharat \\ University of Oxford, Oxford, United Kingdom; \\ tanmay.bharat@path.ox.ac.uk
}

My laboratory studies how molecules on the surface of prokaryotic cells mediate cellular interaction with the environment, enabling cellular motility, initiating cellular adhesion to surfaces, and facilitating biofilm formation. For our work, we leverage our expertise in electron cryotomography (cryo-ET) in situ imaging, together with ongoing method development in subtomogram averaging approaches for structure determination of macromolecules in their native context. We combine cryo-EM with FIB milling of specimens and cryo-light microscopy to study molecules on prokaryotic cells.

Keywords: cryo-EM, cryo-ET, prokaryotes, cellular structural biology 